\title{
Funções de distribuição de probabilidade decendial e mensal para a deficiência hídrica no sol
}

\author{
Probability distribution function ten-day and monthly for soil water deficit
}

\author{
Joel Cordeiro da Silva ${ }^{{ }^{*}}$ Arno Bernando Heldwein ${ }^{\text {II }}$ Gustavo Trentin" ${ }^{\text {III }}$ \\ Nereu Augusto Streck ${ }^{\mathrm{II}}$ Fabrina Bolzan Martins ${ }^{\mathrm{I}}$
}

\section{RESUMO}

A determinação das probabilidades de ocorrência de certos níveis de deficiência hídrica do solo (DHS) é fundamental para o planejamento agropecuário e a gestão ambiental. $O$ objetivo deste trabalho foi determinar as funções de distribuição de probabilidades ( $f d p)$ que melhor caracterizam a distribuição dos valores de deficiência hídrica no solo. As DHSs decendiais e mensais foram determinadas por meio de balanço hídrico diário do solo para as diferentes capacidades de armazenamento de água disponível dos solos da região de abrangência da estação meteorológica de Santa Maria. Verificou-se que os valores mensais e decendiais de DHS se ajustam às funções de distribuição de probabilidades lognormal, gama, exponencial e Weibull. Utilizando-se as fdp selecionadas para cada período do ano, é possível determinar a probabilidade de ocorrência de qualquer valor de DHS para os solos da região de abrangência da estação meteorológica de Santa Maria.

Palavras-chave: balanço hídrico, evapotranspiração, transformação de dados.

\section{ABSTRACT}

The determination of the probability of occurring soil water deficit (DHS) is important for planning agricultural and livestock activities and for environment management. The objective of this study was to determine probability distribution function that fit soil water deficit distribution. Ten-day and monthly DHSs were estimated by calculating daily soil water balance assuming several storage water capacities of soils in the under the scope of the meteorological station of Santa Maria. Monthly and ten-day DHS fit to lognormal, gama, exponential and Weibull probability distribution functions. It is possible to determine the probability of occurring any DHS value at any time throughout the year in the central region of Rio Grande do Sul with the probability distribution functions selected in this study.

Key words: water balance, evapotranspiration, data transformation.

\section{INTRODUÇÃO}

No âmbito da competição de mercado imposta pela globalização, as atividades da agricultura econômica e ecologicamente sustentável precisam ser conduzidas com técnicas precisas, embasadas num sistema de informações dinâmico, por vezes complexo, para que cada tomada de decisão seja a que resulte em menor risco de dano ambiental e econômico (CARDOSO, 2005).

A disponibilidade hídrica no solo (DHS) e a probabilidade de ocorrência de diferentes níveis de deficiência e excesso hídrico são informações de máxima importância, utilizadas na análise de riscos, no zoneamento agrícola e no planejamento de atividades e procedimentos dos empreendimentos agropecuários, bem como na logística do agronegócio. Sendo a disponibilidade hídrica um dos fatores mais importantes da produtividade biológica de um ecossistema natural ou de um cultivo agrícola, a quantificação da sua variabilidade e da probabilidade de ocorrência de determinados níveis críticos é fundamental para o

\footnotetext{
IPrograma de Pós-graduação em Engenharia Agrícola, Universidade Federal de Santa Maria (UFSM), 97105-900, Santa Maria, RS, Brasil. E-mail: joelcordeiro@hotmail.com.*Autor para correspondência.

IIDepartamento de Fitotecnia, Centro de Ciências Rurais (CCR), UFSM, Santa Maria, RS.

IIIPrograma de Pós-graduação em Agronomia, UFSM, Santa Maria, RS, Brasil.
} 
planejamento estratégico. Essas análises só podem ser feitas com a realização do balanço hídrico $(\mathrm{BH})$ específico para cada solo, aplicado de forma seqüencial em séries históricas longas de observações meteorológicas (HELDWEIN et al., 2007).

Para uma adequada determinação de probabilidade de ocorrência de deficiência hídrica, deve-se considerar o método de estimativa de evapotranspiração, a escala de tempo adotada na realização do balanço hídrico, a escolha e a estimativa das funções de distribuição de probabilidade que melhor se ajustam para a deficiência hídrica no solo.

Para analisar a escala de tempo, NIED (2003) trabalhou com balanço hídrico decendial para o solo “São Pedro”, um ARGISSOLO VERMELHO distrófico arênico, visando reavaliar e adequar a distribuição dos dados no tempo em relação ao adotado na estimativa das probabilidades de disponibilidade hídrica publicadas por BURIOL et al. (1980), com base na evapotranspiração de referência (ETo) e no BH seriado mensal, para diferentes valores fixos de capacidade de armazenamento de água disponível no solo (CAD). NIED (2003) verificou que o balanço hídrico pode ser realizado em escala de tempo decendial, desde que a ETo seja calculada em escala diária.

O efeito de diferentes métodos de estimativa da evapotranspiração potencial e as escalas de tempo diário e mensal no cálculo do balanço hídrico foi estudado recentemente por HELDWEIN et al. (2007), para a determinação de probabilidades de deficiências hídricas no solo. Eles concluíram que a ETo mensal estimada pelo método de Thornthwaite, considerando todo o período anual, é significativamente maior do que a totalização mensal dos valores diários de ETo estimados pelo método de Penman e que essa diferença é a principal causa das diferenças de probabilidade de ocorrer deficiência hídrica mensal, quando o $\mathrm{BH}$ seqüencial também é realizado nessa escala de tempo. Em função disso, eles consideraram mais adequado o método de Penman no cálculo da ETo para a determinação de deficiência hídrica no solo e suas probabilidades associadas.

Em relação à escolha e à estimativa das funções de distribuição de probabilidade para a deficiência hídrica no solo, há poucos estudos. Porém, considerando-se que a deficiência hídrica do solo é condicionada pela relação entre a precipitação e a evapotranspiração e pela CAD, que é altamente dependente das características físicas do solo, supõese que a distribuição dos valores de deficiência hídrica do solo apresente semelhança à distribuição dos valores de precipitação. Para analisar a distribuição de dados de precipitação, a função gama tem sido a mais comum (ASSIS, 1991, CASTRO, 1994, RIBEIRO \& LUNARDI, 1997, WILKS, 1999, CASTELLVÍetal., 2004). Além disso, é possível utilizar outras funções de distribuição de probabilidade (fdp) com melhor ajuste do que a função gama, pois há variação na distribuição dos valores de precipitação, conforme o período de tempo utilizado para a separação dos dados, conforme o tipo ou o conjunto de dados (médio, total, absoluto, máximo, mínimo) e conforme o regime pluviométrico do local em estudo (CATALUNHA et al., 2002).

O objetivo deste trabalho foi determinar as fdp que melhor caracterizam a distribuição dos valores decendiais e mensais de deficiência hídrica no solo para as diferentes capacidades de armazenamento de água disponível (CAD) dos solos da região central do Rio Grande do Sul.

\section{MATERIAL E MÉTODOS}

O trabalho foi desenvolvido para a região central do Rio Grande do Sul (RS), na área de abrangência da Estação Meteorológica Principal de Santa Maria. A área estudada localiza-se entre as coordenadas de 29²3'15' e 30²2'17' S e 54 32'07' e $53^{\circ} 05^{\prime} 20^{\prime} \mathrm{W}$, abrangendo uma superfície total de $6640 \mathrm{~km}^{2}$ (CARDOSO, 2005).

Na região de estudo, a precipitação é bem distribuída ao longo do ano (primavera 26\%, verão $24 \%$, outono $25 \%$ e inverno $25 \%$ ), porém, BERLATO (1992) afirma que, embora a precipitação pluvial seja bem distribuída em todas as estações do ano, ocorre grande variabilidade inter-anual desse elemento meteorológico e, conforme BURIOL et al. (1980), as deficiências hídricas são mais freqüentes e mais intensas durante os meses de novembro à março.

Neste estudo foram incluídos os solos mais representativos na área de abrangência da Estação Meteorológica Principal de Santa Maria. Os solos da região podem ser distribuídos nas seguintes classes e ocupação da área: Alissolos (2,16\% da área); Argissolos (42,69\% da área); Chernossolos (5,81\% da área); Latossolos (0,57\% da área), Luvissolos (4,50\% da área); Neossolos (3,14\% da área) e Planossolos (41,13\% da área) (CARDOSO, 2005). A determinação da capacidade de armazenamento de água disponível (CAD) foi realizada para condições de vegetação nativa, sendo consideradas as características físicas do perfil e a profundidade de ocorrência significativa de raízes, conforme BRASIL (1973). Foram utilizados os valores de CAD de 40, 60, 95 104, 120, 135 e 215mm calculado para todos os tipos de solo da região de estudo (Tabela 1). Mais detalhes sobre as características físicas dos solos relacionadas à sua CAD e sobre os parâmetros 
Tabela 1 - Capacidade de armazenamento de água disponível no solo (CAD), unidades de mapeamento e classificação dos solos da área de abrangência da estação meteorológica principal de Santa Maria, RS, (BRASIL, 1973; PERAZA, 2003).

\begin{tabular}{lll}
\hline CAD $(\mathrm{mm})$ & Unidades de mapeamento (BRASIL, 1973) & Classificação dos solos (EMBRAPA, 1999) \\
\hline 40 & Charrua & Neossolo Litólico Eutrófico Chernozênico \\
40 & Ciriaco & Chernossolo Argilúvico férrico típico \\
40 & Guassupi & Neossolo Litólico Distrófico típico \\
60 & Júlio de Castilhos & Argissolo vermelho-amarelo alumínico típico \\
95 & Venda Grande & Chernossolo Argilúvico órtico saprolítico \\
104 & Santa Maria & Alissolo Hipocrômico argilúvico típico \\
104 & Vila & Chernossolo Háplico órtico típico \\
120 & Oásis & Argissolo vermelho-amarelo alumínico alissólico \\
120 & Piraí & Luvissolo Hipocrômico órtico típico \\
120 & São Gabriel & Planossolo Háplico Eutrófico típico \\
120 & São Pedro & Argissolo vermelho distrófico arênico \\
135 & Alto das Canas & Argissolo vermelho distrófico latossólico \\
135 & Cambai & Luvissolo Crômico órtico típico \\
135 & Vacacaí & Planossolo Hidromórfico Eutrófico Arênico \\
215 & Cerrito & Latossolo Vermelho Distrófico Argissólico \\
\hline
\end{tabular}

da função de VAN GENUCHTEN (1980) utilizados na determinação dos valores de CAD podem ser obtidos em PERAZA (2003) e CARDOSO (2005).

O balanço hídrico $(\mathrm{BH})$ do solo foi calculado por meio da evapotranspiração diária calculada pelo método de Penman (ALLEN et al., 1998), dos valores diários da precipitação e da CAD para cada tipo de solo. Os cálculos foram realizados para o período da série histórica do banco de dados da Estação Meteorológica Principal de Santa Maria, coletados de julho de 1968 a julho de 2006, conforme metodologia proposta por THORNTHWAITE \& MATHER (1955), NIED (2003) e SILVA(2005).

Por meio do cálculo do BH, foram obtidos os valores diários de deficiência hídrica do solo (DHS), os quais foram somados obtendo-se o valor da deficiência hídrica total acumulada por período, considerando-se os valores de deficiência diária que ocorreram somente durante o período em questão (mês ou decêndio). Também foram obtidas as deficiências máximas acumuladas, que, nesse caso, representam os valores máximos de deficiência hídrica no solo, obtidos pela soma dos valores diários de deficiência a partir do início da ocorrência de cada período contínuo de deficiência hídrica, mesmo que este iniciasse em qualquer data anterior ao período considerado.

Os valores de deficiência hídrica máxima (DHM) e de deficiência hídrica total (DHT), obtidos para cada mês ou decêndio e para cada valor de CAD, foram submetidos à análise de distribuição de probabilidade e a teste para qualidade de ajuste das funções, para verificar qual fdp melhor representa a distribuição dos valores de deficiência hídrica no solo em cada período considerado. Também foram testadas transformações (raiz quadrada e raiz cúbica) nos dados de deficiência para verificar a hipótese de melhor ajuste das funções à distribuição dos dados.

Em uma primeira análise, feita visualmente, pela plotagem da distribuição de dados, foram escolhidas as funções que poderiam apresentar ajustes aos dados. Foram então testados os ajustes das fdp: exponencial, gama, lognormal e Weibull, reduzidas a dois parâmetros, conforme preconizam CAMPOS (1979), ASSIS et al. (1996) e CATALUNHA et al. (2002). Após o ajuste, todas as fdp foram selecionadas por meio dos testes de Chi-Quadrado e KolmogorovSmirnov (CAMPOS, 1979; D’AGOSTINO \& STEPHENS, 1986; ASSIS et al., 1996). Como critério inicial, foram selecionados as fdp em que o valor de probabilidade, para as estatísticas calculadas, de pelo menos um dos testes citados foi maior do que o nível de significância de $10 \%$, sendo que as demais foram descartadas. Os testes de ajuste de distribuição de probabilidade analisados consideram como hipótese nula aquela em que não há diferença entre a freqüência relativa empírica e a freqüência relativa teórica, definida matematicamente (CARGNELUTTI FILHO et al., 2004). Dessa forma, foram testadas, separadamente, as quatro fdp ajustadas para os 38 valores (38 anos de dados meteorológicos) de deficiência hídrica no solo (máxima, total e suas transformações), para as sete CAD dos diferentes tipos de solo e para os 12 meses ou 36 decêndios do ano, totalizando 8064 combinações, sendo 2016 combinações para DHS mensal e 6048 para DHS decendial. 


\section{RESULTADOS E DISCUSSÃO}

Por meio da análise de distribuição, foi possível o ajuste de pelo menos uma fdp para quase todas CAD e todos períodos do ano. Considerando-se as funções testadas para todas as combinações possíveis, houve predominância da função lognormal, seguida da função gama, Weibull e exponencial (Tabela 2).

A transformação dos dados, utilizando-se raiz quadrada e raiz cúbica, foi eficiente para melhorar o ajuste de praticamente todas as fdp, exceto para a função exponencial (Tabela 2). A transformação dos dados pela raiz cúbica resultou em maior número de casos em que o ajuste das fdp foi significativo um nível de $10 \%$ de probabilidade de erro pelo teste de $\chi^{2}$, em relação à transformação utilizando-se a raiz quadrada para a deficiência hídrica máxima decendial, máxima mensal e total mensal. Para a deficiência hídrica total decendial, o ajuste pela raiz quadrada resultou em um número pouco maior de casos significativos (Tabela 2), em um nível de $10 \%$ de probabilidade de erro pelo teste de $\chi^{2}$, nível de probabilidade de erro usual em testes de aderência (ASSIS et al., 1996; CARGNELUTTI FILHO et al., 2004).
O ajuste dos dados pela raiz quadrada e pela raiz cúbica provoca o aumento dos valores maiores do que zero e menores do que $1 \mathrm{~mm}$. Esses valores de DHS de baixa intensidade foram freqüentes principalmente quando o BH estava na escala decendial, para solos com alta $\mathrm{CAD}(104,120$ e 215mm). Devido à alta retenção de água no solo, os solos com alta CAD possuem uma maior capacidade de suprir a água necessária para atender à demanda atmosférica, enquanto que outros solos com menor CAD poderão esgotar suas reservas em períodos curtos de estiagem, portanto, a DHS inicia antes e é mais intensa nos solos com menor CAD. Por essa razão, períodos curtos (menores que cinco dias) com DHS menores do que $1 \mathrm{~mm}$ são mais freqüentes em solos com alta CAD, enquanto que para os solos com baixa CAD ocorrem DHS mais intensas e prolongadas. Portanto, é esperado que esse tipo de transformação dos dados seja mais eficiente, no sentido de melhorar o ajuste das fdp, quando o BH é realizado em escala decendial, para solos com alta CAD.

O "deslocamento" dos dados para a direita, quando foi utilizado o ajuste dos dados pela raiz quadrada ou raiz cúbica, provocou o aumento da moda para próximo de $1 \mathrm{~mm}$ e mudança no padrão (ou forma)

Tabela 2 - Freqüência relativa (\%) de casos em que os totais e os valores máximos acumulados de deficiência hídrica (DH), raiz quadrada (DH2) e de raiz cúbica da deficiência hídrica (DH3), decendiais e mensais, em cada classe de p-valor do teste de aderência do Chi-Quadrado* para as funções de distribuição de probabilidade exponencial, gama, lognormal e Weibull.

\begin{tabular}{|c|c|c|c|c|c|c|c|c|c|c|c|c|}
\hline \multirow[b]{2}{*}{ p-valor } & \multicolumn{3}{|c|}{-----Exponencial----- } & \multicolumn{3}{|c|}{--------Gama-------- } & \multicolumn{3}{|c|}{------Lognomal------ } & \multicolumn{3}{|c|}{------Weibull------ } \\
\hline & $\mathrm{DH}$ & DH2 & DH3 & $\mathrm{DH}$ & DH2 & DH3 & $\mathrm{DH}$ & DH2 & DH3 & $\mathrm{DH}$ & DH2 & DH3 \\
\hline \multicolumn{13}{|c|}{------------------------------------------------------ deficiência hídrica máxima decendial ------------------------------------------------------- } \\
\hline $\mathrm{p}<0,01$ & 84,91 & 21,43 & 86,90 & 69,84 & 42,57 & 20,66 & 28,10 & 16,12 & 7,03 & 58,26 & 45,45 & 36,78 \\
\hline $\mathrm{p}<0,05$ & 4,37 & 19,44 & 9,13 & 9,50 & 8,26 & 12,81 & 19,01 & 7,85 & 8,26 & 13,64 & 11,16 & 13,22 \\
\hline $\mathrm{p}<0,1$ & 2,78 & 15,48 & 2,38 & 6,20 & 7,02 & 10,74 & 14,88 & 12,81 & 10,74 & 6,61 & 4,55 & 4,13 \\
\hline $\mathrm{p}=0,1$ & 7,94 & 43,65 & 1,59 & 14,46 & 42,15 & 55,79 & 38,01 & 63,22 & 73,97 & 21,49 & 38,84 & 45,87 \\
\hline & & & & -- & iciência & Arica tot & decendir & & & & & -- \\
\hline $\mathrm{p}<0,01$ & 17,86 & 65,08 & 100,00 & 18,78 & 4,08 & 3,67 & 7,35 & 2,45 & 3,27 & 11,02 & 4,49 & 4,49 \\
\hline $\mathrm{p}<0,05$ & 14,29 & 17,46 & 0,00 & 24,49 & 8,57 & 8,16 & 15,10 & 11,02 & 7,76 & 22,04 & 9,39 & 10,20 \\
\hline $\mathrm{p}<0,1$ & 9,52 & 7,54 & 0,00 & 10,20 & 7,76 & 9,39 & 11,43 & 6,12 & 10,20 & 14,29 & 12,24 & 13,88 \\
\hline $\mathrm{p}=0,1$ & 58,33 & 9,92 & 0,00 & 46,53 & 79,59 & 78,78 & 66,12 & 80,41 & 78,77 & 52,65 & 73,88 & 71,43 \\
\hline & & & & -- & ciência & rica má & na mens & --------- & & & ---------- & -- \\
\hline $\mathrm{p}<0,01$ & 64,29 & 65,47 & 100,00 & 73,81 & 57,15 & 45,24 & 51,19 & 28,57 & 16,67 & 72,62 & 64,29 & 64,29 \\
\hline $\mathrm{p}<0,05$ & 7,14 & 16,67 & 0,00 & 7,14 & 9,52 & 14,29 & 13,10 & 26,19 & 17,86 & 3,57 & 7,14 & 13,10 \\
\hline $\mathrm{p}<0,1$ & 5,95 & 2,38 & 0,00 & 2,38 & 4,76 & 7,14 & 8,33 & 7,14 & 10,71 & 5,95 & 8,33 & 5,95 \\
\hline $\mathrm{p}=0,1$ & 22,62 & 15,48 & 0,00 & 16,67 & 28,57 & 33,33 & 27,38 & 38,10 & 54,76 & 17,86 & 20,24 & 16,68 \\
\hline & & & & --- & iciênci & ídrica to & mensal & - & & . & -1--- & -- \\
\hline $\mathrm{p}<0,01$ & 11,91 & 92,86 & 100,00 & 44,05 & 17,86 & 14,29 & 20,24 & 3,57 & 7,14 & 41,67 & 23,81 & 25,00 \\
\hline $\mathrm{p}<0,05$ & 19,05 & 7,14 & 0,00 & 13,10 & 11,90 & 8,33 & 9,52 & 14,29 & 9,53 & 13,10 & 20,24 & 19,05 \\
\hline $\mathrm{p}<0,1$ & 10,71 & 0,00 & 0,00 & 8,33 & 9,52 & 9,52 & 13,10 & 8,33 & 8,33 & 10,71 & 19,05 & 11,90 \\
\hline $\mathrm{p}=0,1$ & 58,33 & 0,00 & 0,00 & 34,52 & 60,72 & 67,86 & 57,14 & 73,81 & 75,00 & 34,52 & 36,90 & 44,05 \\
\hline
\end{tabular}

* as funções estatisticamente significativas estão na classe de p-valor maior ou igual a 10\%.

Ciência Rural, v.38, n.7, out, 2008. 
de distribuição dos dados, aproximando-se à forma das curvas lognormal, gama e Weibull. Por essa razão, as transformações citadas foram eficientes para quase todas as fdp, exceto para a função exponencial que possui forte assimetria à esquerda ou forma decrescente da esquerda para a direita, semelhante à distribuição dos dados sem transformação.

Mesmo existindo um grande número de fdps com ajuste significativo em nível de $10 \%$ de probabilidade de erro pelo teste de $\chi^{2}$, houve dificuldades para selecionar uma fdp que tivesse um ajuste estatisticamente significativo para todos os períodos do ano e para todas as CAD, simultaneamente. Portanto, para selecionar as fdp por período do ano (mês ou decêndio), opta-se por aquela que apresentou melhor qualidade do ajustamento (maior valor de " $\mathrm{p}$ " para a estatística do teste do $\chi^{2}$ ), para todas as CAD. Dessa forma, procurou-se selecionar uma fdp representativa para a maioria dos casos. Nas tabelas 3 e 4 podem ser encontrados os parâmetros escalar e de forma (quando possível) das fdp selecionadas para os meses e decêndios do ano e para as CAD dos solos da região central do RS. Nos períodos e para as CADs em que não foi possível o ajuste de nenhuma fdp, não há valor numérico nas referidas tabelas. $\mathrm{O}$ valor do parâmetro de forma da função exponencial também está em branco porque essa função possui apenas um parâmetro, o chamado parâmetro escalar.

Na tabela 3 são apresentados os parâmetros das fdp selecionadas para a deficiência hídrica do solo mensal total e deficiência hídrica do solo mensal máxima. Optou-se, na maioria dos casos, pela função lognormal, utilizando-se o ajustamento da raiz quadrada para DHS mensal total e raiz cúbica para DHS mensal máxima. Nos períodos em que não foi possível ajustar a função lognormal para todas as CADs, optou-se pela função

Tabela 3 - Parâmetros das funções de distribuição de probabilidades (fdp) lognormal (LN) e exponencial (EX), estimadas para as variáveis (V) deficiência hídrica (DH), raiz quadrada da deficiência hídrica (DH2) e raiz cúbica da deficiência hídrica (DH3), para cada mês do ano (M) e para as capacidades de armazenamento de água disponível no solo (CAD) de 40, 95, 120 e 215 mm, para os solos da região central do RS. Dados históricos coletados de julho de 1968 a julho de 2006.

\begin{tabular}{|c|c|c|c|c|c|c|c|c|c|c|}
\hline \multicolumn{3}{|c|}{$-----C A D \rightarrow-----$} & \multicolumn{2}{|c|}{---------40--------- } & \multicolumn{2}{|c|}{---------95--------- } & \multicolumn{2}{|c|}{---------120--------- } & \multicolumn{2}{|c|}{---------215--------- } \\
\hline V & fdp & M & Escalar & Forma & Escalar & Forma & Escalar & Forma & Escalar & Forma \\
\hline \multicolumn{11}{|c|}{----------------------------------------------------------- Deficiência hídrica mensal total ----------------------------------------------------------- } \\
\hline $\mathrm{DH} 2$ & LN & $\mathrm{J}$ & 1,772 & 0,310 & 1,546 & 0,386 & 1,474 & 0,404 & 1,278 & 0,441 \\
\hline DH2 & $\mathrm{LN}$ & $\mathrm{F}$ & 1,490 & 0,324 & 1,217 & 0,404 & & & & \\
\hline DH3 & $\mathrm{LN}$ & $\mathrm{M}$ & 0,984 & 0,201 & 0,800 & 0,252 & 0,748 & 0,267 & 0,610 & 0,300 \\
\hline DH2 & $\mathrm{LN}$ & A & 1,028 & 0,397 & 0,708 & 0,470 & 0,622 & 0,489 & 0,408 & 0,506 \\
\hline DH2 & $\mathrm{LN}$ & $\mathrm{M}$ & 0,446 & 0,383 & 0,100 & 0,476 & & & $-0,256$ & 0,547 \\
\hline DH2 & $\mathrm{LN}$ & $\mathrm{J}$ & $-0,099$ & 0,436 & $-0,505$ & 0,456 & $-0,620$ & 0,462 & $-0,914$ & 0,486 \\
\hline DH2 & $\mathrm{LN}$ & $\mathrm{J}$ & 0,100 & 0,399 & $-0,313$ & 0,411 & $-0,428$ & 0,414 & $-0,719$ & 0,422 \\
\hline DH2 & $\mathrm{LN}$ & A & 0,694 & 0,331 & 0,306 & 0,354 & 0,196 & 0,358 & $-0,084$ & 0,365 \\
\hline DH2 & $\mathrm{LN}$ & S & 1,032 & 0,305 & 0,672 & 0,350 & 0,567 & 0,359 & 0,296 & 0,373 \\
\hline DH2 & $\mathrm{LN}$ & $\mathrm{O}$ & 1,340 & 0,367 & 1,004 & 0,422 & 0,904 & 0,433 & 0,642 & 0,452 \\
\hline DH2 & $\mathrm{LN}$ & $\mathrm{N}$ & 1,632 & 0,345 & 1,332 & 0,421 & 1,238 & 0,436 & 0,989 & 0,465 \\
\hline DH2 & $\mathrm{LN}$ & $\mathrm{D}$ & 1,847 & 0,239 & 1,623 & 0,314 & 1,548 & 0,334 & 1,335 & 0,376 \\
\hline-- & & & & ------- I & ência híd & hensal m & 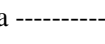 & & & ------- \\
\hline DH3 & $\mathrm{LN}$ & $\mathrm{J}$ & 1,065 & 0,304 & 0,909 & 0,345 & 0,856 & 0,355 & 0,710 & 0,376 \\
\hline $\mathrm{DH}$ & $\mathrm{EX}$ & $\mathrm{F}$ & 16,560 & & 10,981 & & $2,694^{\mathrm{I}}$ & & $2,244^{\mathrm{I}}$ & \\
\hline DH3 & $\mathrm{LN}$ & $\mathrm{M}$ & 0,827 & 0,271 & 0,630 & 0,308 & 0,574 & 0,320 & 0,436 & 0,343 \\
\hline DH3 & $\mathrm{LN}$ & A & 0,608 & 0,349 & 0,385 & 0,386 & 0,326 & 0,396 & 0,184 & 0,394 \\
\hline DH3 & $\mathrm{LN}$ & $\mathrm{M}$ & 0,174 & 0,342 & $-0,056$ & 0,392 & $-0,122$ & 0,406 & $-0,296$ & 0,433 \\
\hline DH3 & $\mathrm{LN}$ & $\mathrm{J}$ & $-0,207$ & 0,369 & $-0,479$ & 0,380 & $-0,554$ & 0,382 & $-0,743$ & 0,388 \\
\hline DH3 & $\mathrm{LN}$ & $\mathrm{J}$ & $-0,101$ & 0,316 & $-0,375$ & 0,323 & $-0,450$ & 0,324 & $-0,643$ & 0,329 \\
\hline DH3 & $\mathrm{LN}$ & A & 0,305 & 0,263 & 0,047 & 0,276 & $-0,026$ & 0,278 & $-0,212$ & 0,282 \\
\hline DH3 & $\mathrm{LN}$ & S & 0,543 & 0,284 & 0,302 & 0,309 & 0,232 & 0,313 & 0,051 & 0,321 \\
\hline DH3 & $\mathrm{LN}$ & $\mathrm{O}$ & 0,710 & 0,332 & 0,480 & 0,361 & 0,412 & 0,367 & 0,236 & 0,377 \\
\hline DH3 & $\mathrm{LN}$ & $\mathrm{N}$ & 0,913 & 0,329 & 0,710 & 0,377 & 0,647 & 0,386 & 0,479 & 0,404 \\
\hline DH3 & $\mathrm{LN}$ & $\mathrm{D}$ & 1,095 & 0,264 & 0,927 & 0,303 & 0,874 & 0,312 & 0,727 & 0,331 \\
\hline
\end{tabular}

\footnotetext{
${ }^{1}$ Para a deficiência hídrica máxima, no mês de fevereiro e CAD de 120 e 215 mm, foi selecionada a fdp exponencial com a variável transformada pela raiz quadrada.
} 
Tabela 4 - Parâmetros das funções de distribuição de probabilidades (fdp) exponencial (EX), gama (GM) e lognormal (LN) estimadas para as variáveis (V) de deficiência hídrica máxima na forma transformada pela raiz quadrada (DH2) e raiz cúbica (DH3), para cada decêndio do ano (D) e para as capacidades de armazenamento de água disponível (CAD) de 40, 95, 120 e 215 mm, para os solos da região central do RS. Dados históricos coletados de julho de 1968 a julho de 2006.

\begin{tabular}{|c|c|c|c|c|c|c|c|c|c|c|c|}
\hline \multicolumn{4}{|c|}{------------CAD $\rightarrow$------------ } & \multicolumn{2}{|c|}{--------40-------- } & \multicolumn{2}{|c|}{-------95------- } & \multicolumn{2}{|c|}{--------120-------- } & \multicolumn{2}{|c|}{--------215-------- } \\
\hline V & fdp & M & $\mathrm{D}$ & Escalar & Forma & Escalar & Forma & Escalar & Forma & Escalar & Forma \\
\hline DH3 & $\mathrm{LN}$ & Jan & 1 & 0,851 & 0,394 & 0,683 & 0,446 & 0,629 & 0,457 & 0,494 & 0,468 \\
\hline DH3 & $\mathrm{LN}$ & & 2 & 0,770 & 0,452 & 0,612 & 0,484 & 0,562 & 0,494 & 0,434 & 0,501 \\
\hline DH3 & $\mathrm{LN}$ & & 3 & 0,797 & 0,382 & 0,620 & 0,426 & 0,566 & 0,435 & 0,430 & 0,447 \\
\hline DH3 & $\mathrm{LN}$ & Fev & 1 & 0,548 & 0,372 & 0,371 & 0,417 & 0,320 & 0,433 & 0,196 & 0,459 \\
\hline DH3 & $\mathrm{LN}$ & & 2 & 0,537 & 0,435 & 0,342 & 0,474 & 0,294 & 0,485 & 0,159 & 0,503 \\
\hline & & & $3^{I}$ & & & & & & & & \\
\hline DH3 & GM & Mar & 1 & 0,255 & 7,573 & 0,264 & 6,126 & 0,269 & 5,710 & 0,274 & 4,962 \\
\hline DH3 & $\mathrm{LN}$ & & 2 & 0,541 & 0,463 & 0,331 & 0,508 & 0,276 & 0,519 & 0,138 & 0,519 \\
\hline DH3 & $\mathrm{LN}$ & & 3 & 0,548 & 0,336 & 0,365 & 0,346 & 0,314 & 0,347 & 0,170 & 0,366 \\
\hline DH2 & $\mathrm{LN}$ & Abr & 1 & 0,601 & 0,802 & 0,259 & 0,858 & 0,172 & 0,875 & $-0,035$ & 0,887 \\
\hline DH3 & $\mathrm{LN}$ & & 2 & 0,188 & 0,518 & $-0,020$ & 0,553 & $-0,076$ & 0,566 & $-0,228$ & 0,592 \\
\hline DH3 & $\mathrm{LN}$ & & 3 & 0,135 & 0,398 & $-0,085$ & 0,433 & $-0,150$ & 0,443 & $-0,302$ & 0,454 \\
\hline DH3 & $\mathrm{LN}$ & Set & 1 & 0,242 & 0,415 & $-0,012$ & 0,437 & $-0,084$ & 0,441 & $-0,269$ & 0,447 \\
\hline DH3 & $\mathrm{LN}$ & & 2 & 0,160 & 0,515 & $-0,089$ & 0,536 & $-0,161$ & 0,539 & $-0,344$ & 0,546 \\
\hline DH3 & $\mathrm{LN}$ & & 3 & 0,274 & 0,387 & 0,024 & 0,409 & $-0,046$ & 0,414 & $-0,228$ & 0,422 \\
\hline DH3 & $\mathrm{LN}$ & Out & 1 & 0,281 & 0,409 & 0,035 & 0,432 & $-0,034$ & 0,436 & & \\
\hline DH3 & $\mathrm{LN}$ & & 2 & 0,501 & 0,420 & 0,268 & 0,446 & 0,200 & 0,452 & 0,021 & 0,464 \\
\hline DH3 & $\mathrm{LN}$ & & 3 & 0,582 & 0,420 & 0,359 & 0,452 & 0,293 & 0,458 & 0,118 & 0,469 \\
\hline DH3 & $\mathrm{LN}$ & Nov & 1 & 0,503 & 0,395 & 0,272 & 0,440 & 0,204 & 0,448 & 0,030 & 0,463 \\
\hline DH3 & $\mathrm{LN}$ & & 2 & 0,722 & 0,348 & 0,505 & 0,390 & 0,439 & 0,399 & 0,267 & 0,415 \\
\hline DH3 & $\mathrm{LN}$ & & 3 & 0,798 & 0,422 & 0,601 & 0,463 & 0,540 & 0,471 & 0,375 & 0,486 \\
\hline DH3 & $\mathrm{LN}$ & Dez & 1 & 0,814 & 0,395 & 0,650 & 0,433 & 0,593 & 0,443 & 0,437 & 0,461 \\
\hline DH3 & $\mathrm{LN}$ & & 2 & 0,806 & 0,462 & 0,665 & 0,445 & 0,616 & 0,443 & 0,473 & 0,450 \\
\hline DH3 & $\mathrm{LN}$ & & 3 & 0,866 & 0,448 & 0,692 & 0,495 & 0,640 & 0,507 & 0,500 & 0,526 \\
\hline
\end{tabular}

${ }^{1}$ Não foi possível selecionar nenhuma fdp para o terceiro decêndio do mês de fevereiro.

com ou sem transformação da variável estudada. Não foi possível ajustar nenhuma fdp para a DHS mensal total, para os meses de fevereiro, CAD de 120 e 215 mm, e para o mês de maio, CAD de $120 \mathrm{~mm}$.

Os parâmetros das fdp selecionadas para a DHS máxima decendial encontram-se na tabela 4. Afdp predominante foi a função lognormal, com transformação de raiz cúbica. Também houve dois decêndios em que foi ajustada a função gama, com a transformação pela raiz cúbica e um decêndio com a função exponencial. Para qualquer CAD no terceiro decêndio de fevereiro, para a CAD de $104 \mathrm{~mm}$ no primeiro decêndio de julho, e para o primeiro decêndio de outubro e CAD de 215mm, também não foi possível ajustar nenhuma fdp.

O risco de deficiência hídrica decendial na metade sul do Estado do Rio Grande do Sul a partir da freqüência empírica foi determinado por LEIVAS et al. (2006), sendo que eles não encontraram nenhuma fdp para a variável que utilizaram como medida da deficiência hídrica. LEIVAS et al. (2006) utilizaram uma metodologia semelhante à utilizada no presente trabalho. No entanto, como indicador de deficiência hídrica, eles utilizaram o índice de satisfação das necessidades de água (ISNA), que representa o quociente entre a evapotranspiração real e a evapotranspiração de referência (ETR ETo-1 ${ }^{-1}$. O motivo para não terem encontrado nenhuma fdp provavelmente se deve ao fato de que LEIVAS et al. (2006) utilizaram o balanço hídrico em escala decendial, ao invés de escala diária com separação decendial dos dados, e utilizaram o índice $\mathrm{R}$, que varia entre zero e um, reduzindo, dessa forma, o número $(\mathrm{N})$ de dados e com pouca amplitude, o que dificulta o ajuste das fdps.

A distribuição dos dados de deficiência hídrica do solo se assemelha à distribuição dos dados de precipitação. No entanto, há maior freqüência para os valores próximos de zero, o que torna a distribuição dos dados de deficiência hídrica do solo mais semelhante à forma da distribuição lognormal ou exponencial, enquanto que para os dados de 
precipitação (SILVA et al., 2007) verificaram que as fdps com melhor ajuste foram gama e Weibull.

\section{CONCLUSÕES}

As funções que predominam para caracterizar a distribuição dos dados mensais de deficiência hídrica do solo de diferentes CAD são as funções de distribuição lognormal e exponencial, com melhor ajuste quando utiliza-se a transformação nos dados pela raiz cúbica e raiz quadrada.

Para a deficiência hídrica decendial, predominam as funções lognormal, exponencial e gama, com melhor ajuste quando são utilizadas as transformações nos dados pela raiz cúbica e raiz quadrada.

\section{REFERÊNCIAS}

ALLEN, R.G. et al. Crop evapotranspiration guidelines for computing crop water requirements. Roma: FAO Irrigation and Drainage Paper, 1998. 328p. (Boletim Técnico, 56).

ASSIS, F.N. et al. Aplicações de estatísticas à climatologia: teoria e prática. Pelotas: Ufpel, 1996. 161p.

ASSIS, F.N. de Modelagem da ocorrência e da quantidade de chuva e de dias secos em Piracicaba - SP e Pelotas RS. 1991. 134f. Tese (Doutorado em Agronomia) - Escola Superior de Agricultura Luiz de Queiroz, Universidade de São Paulo.

BERLATO, M.A. As condições de precipitação pluvial no Estado do Rio Grande do Sul e os impactos da estiagem na produção agrícola. In: BERGAMASCHI, $H$. et al Agrometeorologia aplicada à irrigação. Porto Alegre: UFRGS, 1992. p.11-23.

BURIOL, G.A. et al. Disponibilidades hídricas do solo possíveis de ocorrerem no estado do Rio Grande do Sul. Revista do Centro de Ciências Rurais, v.10 (suplemento), p.1-141, 1980.

BRASIL. Levantamento de reconhecimento dos solos do estado do Rio Grande do Sul. Recife: Ministério da Agricultura, 1973. 431p. (Boletim Técnico, 30).

CAMPOS, H. Estatística experimental não paramétrica. Piracicaba: Esalq, 1979. 343p.

CARGNELUTTI FILHO, A. et al. Ajustes de funções de distribuição de probabilidade à radiação solar global no Estado do Rio Grande do Sul. Pesquisa Agropecuária Brasileira, v.39, n.12, p.1157-1166, 2004.

CARDOSO, C.D.V. Probabilidade de ocorrência de deficiência hídrica nos solos da região Central do Estado do Rio Grande do Sul. 2005. 162f. Tese (Doutorado em Agronomia) - Curso de Pós-graduação em Agronomia, Universidade Federal de Santa Maria.

CASTELLVÍ, F. et al. Generation of daily amounts of precipitation from standard climatic data: a case study for Argentina. Journal of Hydrology, v.289, n.4, p.286-302, 2004.

CASTRO, R. Distribuição probabilística da freqüência de precipitação na região de Botucatu, SP. 1994. 101f. Dissertação (Mestrado em Agronomia) - Universidade Estadual Paulista.

CATALUNHA, M.J. et al. Aplicação de cinco funções densidade de probabilidade a séries de precipitação pluvial no Estado de Minas Gerais. Revista Brasileira de Agrometeorologia, v.10, n.1, p.153-162, 2002

D’AGOSTINO, R.B.; STEPHENS, M.A. Goodness-of-fit techniques. New York: Marcel Dekker, 1986. 286p.

EMBRAPA. Centro Nacional de Pesquisa em Solos. Sistema Brasileiro de Classificação de solos. Brasília: Embrapa, 1999. 412p

HELDWEIN, A.B. et al. Probabilidade de ocorrência de deficiência hídrica no centro do Rio Grande do Sul em função do método de cálculo da evapotranspiração e da escala de tempo do balanço hídrico. Revista Brasileira de Agrometeorologia, 2007. (em trâmite)

LEIVAS, J.F. et al. Risco de deficiência hídrica decendial na metade sul do Estado do Rio Grande do Sul. Revista Brasileira de Engenharia Agrícola e Ambiental, v.10, n.2, p.397407, 2006

NIED, A.H. Balanço hídrico diário do solo simulado para diferentes épocas de semeadura do milho em Santa Maria, RS. 2003. 73f. Dissertação (Mestrado em Agronomia) - Curso de Pós-graduação em Agronomia, Universidade Federal de Santa Maria.

PERAZA, J.E.S. Retenção de água e pedofunções para solos do Rio Grande do Sul. 2003. 117f. Dissertação (Mestrado Engenharia Agrícola) - Curso de Pós-graduação em Engenharia Agrícola, Universidade Federal de Santa Maria.

RIBEIRO, A.M.; LUNARDI, D.M.C. A precipitação mensal provável para Londrina - PR, através da função gama. Energia na Agricultura, v.12, n.4, p.37-44, 1997.

SILVA, J.C. da. Épocas de menor risco de estresse hídrico e térmico para o feijoeiro na região central do Rio Grande do Sul. 2005. 64f. Dissertação (Mestrado em Engenharia Agrícola) - Curso de Pós-graduação em Engenharia Agrícola, Universidade Federal de Santa Maria.

SILVA, J.C. da et al. Análise de distribuição de chuva para Santa Maria, RS. Revista Brasileira de Engenharia Agrícola e Ambiental, v.11, n.1, p.67-72, 2007.

THORNTHWAITE, C.W.; MATHER, J.R. The water balance Centerton: Institute of Tecnology, Laboratory of Climatology, 1955. 104p.

VAN GENUCHTEN, M.T. A closed-form equation for predicting the hydraulic conductivity of unsaturated soils. Soil Science Society of American Journal, v.44, p.892-898, 1980.

WILKS, D.S. Interannual variability and extreme-value characteristics of several stochastic daily precipitation models. Agricultural and Forest Meteorology, v.93, n.3, p.153169, 1999. 